\title{
A Platinum Resistance Bridge Thermometer
}

\author{
Ximin Liu \\ School of Electrical Engineering, University of Jinan, Jinan City, China, 250022 \\ cse_Ixm@ujn.edu.cn
}

Keywords: Pt100. Bridge Circuit. Linear Temperature Measurement. Reference Table

Abstract.A platinum resistance bridge thermometer is introduced. It implements linear temperature measurement in -200 850 degrees with a bridge circuit. It consists of bridge circuit, multi-channel switches, amplifier, AD574, display, COM port, precision power supply, 89C51 etc. After the analysis of bridge circuit characteristic, a method is presented that the platinum resistance value is obtained by computing the current ratio of tow bridge circuit loop and then looking into the Pt100 reference table to obtain the measured temperature. So the linear temperature measurement is implemented without nonlinear compensation. The measurement error is less than 1.5 degrees.

\section{Introduction}

It is a most widely used method to measure the thermal resistance using a bridge circuit. In addition to the balanced bridge circuit used in laboratory the bridge circuit used to realize continuous measurement is work mostly in unbalanced state. It is a nonlinear relationship between the output voltage of the bridge circuit and the measured resistance. Generally we use a approximate linear method to complete the resistance measurement. This bring measurement error. The larger the measurement range the more the measurement error, sometimes up to $3 \sim 5 \%$. It is very adverse for measurement accuracy. The measurement error will be larger considering the nonlinearity between the platinum resistance and temperature. For this point there are many nonlinear compensation methods by software or hardware technology that improved the measurement accuracy to a certain extent $^{[1-5]}$. This paper presents a simple and practical method to measure the thermal resistance with a bridge circuit. The linear temperature measurement can be realized without nonlinear compensation.

\section{Bridge Circuit}

Single-arm Bridge Circuit. The simplest single-arm bridge circuit is shown in Fig. $1 . R_{t}$ is the measured resistance, $R_{1}, R_{2}, R_{3}$ is fixed resistors, $E$ is power supply, $U_{i}$ is output, $I_{1}, I_{2}$ is the loop current of the two bridge loop at left and right. $U_{p}$ is the voltage of the point a in left two arm branch and its value changes with $R_{t}, U_{\mathrm{s}}$ is the voltage of the point $\mathrm{b}$ in right two arm branch and its value is a constant. The output voltage $U_{i}$ is

$$
U_{i}=U_{\mathrm{s}}-U_{p}=\frac{R_{2}}{R_{1}+R_{2}} E-\frac{R_{3}}{R_{3}+R_{t}} E=\frac{R_{2} R_{\mathrm{t}}-R_{1} R_{3}}{\left(R_{1}+R_{2}\right)\left(R_{3}+R_{t}\right)} E
$$

It is nonlinearity between $U_{i}$ and $R_{t}$. Generally, let $R_{t}=R_{\mathrm{t} 0}+\Delta R_{t}, R_{1}=R_{2}=R_{3}=R_{\mathrm{t} 0}=R$, Eq. 1 simplified as

$$
U_{i}=\frac{\Delta R_{t}}{4 R\left(1+\frac{\Delta R_{t}}{2 R}\right)} E
$$

Eq. 2 is the precise characteristic of $U_{i}$ and $R_{t}$. when $\Delta R_{t}<<R_{t 0}=R$, ignore $\frac{\Delta R_{t}}{2 R}$, Eq. 2 approximation as

$$
U_{i}=\frac{\Delta R_{t}}{4 R} E
$$

Eq. 3 is a approximate linear relationship between $U_{i}$ and $\Delta R_{t}$ when $\Delta R_{t}<<R_{\mathrm{t} 0}$. It is the approximate characteristic of $U_{i}$ and $R_{t}$. Let $\Delta R_{t}$ in $0 \sim 400 \Omega$, when $R=1 \mathrm{k} \Omega, 2 \mathrm{k} \Omega, 3 \mathrm{k} \Omega$, the curve of Eq. 2 and Eq. 3 is shown in Fig. 2. It is clear that the greater the $R$ the smaller the error between Eq.2 and Eq. 3, and $U_{i}$ range is reduced at the same time. The approximate value is greater than the precise value under the same $\Delta R_{t}$. When $R=3 \mathrm{k} \Omega, \Delta R_{t}=290 \Omega$, the error is $+4.6 \%$ for the approximate value. For Pt 100 the 


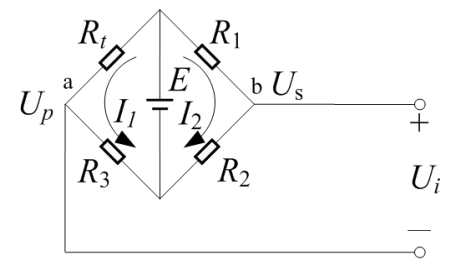

Figure 1. single-arm bridge circuit

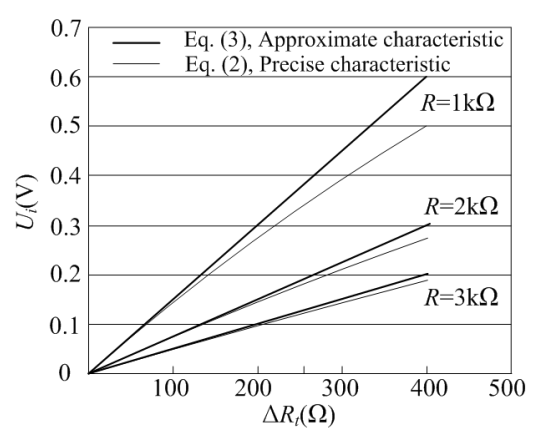

Figure 2. The curve of precise characteristic and approximate characteristic with different $R$ ${ }^{\circ} \mathrm{C}$

corresponding temperature error is about 35 . The error will be larger coupled with the nonlinear characteristic of platinum resistance itself. Many nonlinear compensation methods was adopted to fix this error in instruments. This error include two aspects, one is the approximation error when using Eq. 3 , the other is the inherent nonlinear error of platinum resistance. The two errors correction is tedious.

Platinum Resistance Bridge Circuit It is very easy to calculate the measured resistance $\Delta R_{t}$ with Eq. 2 based on microprocessor after measuring the $U_{i}$. But Eq. 2 is related to the power supply $E$ and many factors can influence the stability of $E$, that is very bad for accurate measurement. In addition, it is necessary to do compensation for the impact of lead resistance when using platinum resistance to measure temperature. Usually use a three-wire connection. The improved bridge circuit is shown in Fig. 3. Here $R_{t}$ is Pt100. The $r$ is equivalent resistance of the three leads of $R_{t}$ and its maximum value

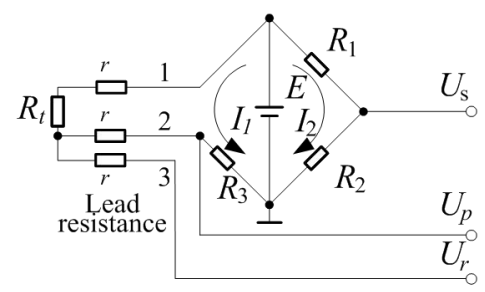

Figure 3. Platinum resistance bridge circuit

is $15 \Omega$. On both ends of $R_{t}$ the lead 1 and 2 connected to the upper and lower arm of the bridge circuit left side respectively. Another lead 3 is used as a output terminal for $U_{r} . U_{r}$ is compensation voltage signal of lead resistance. For the left loop, there is

$$
E=\left(r+R_{t}+r+R_{3}\right) I_{1}
$$

Also, For the right loop, there is

$$
E=\left(R_{1}+R_{2}\right) I_{2}
$$

Because Eq. $4=$ Eq. 5 . there is

$$
R_{t}=\left(R_{1}+R_{2}\right) \frac{I_{2}}{I_{1}}-2 r-R_{3}
$$

Eq. 6 is the computing equation to realize the Pt100 measurement with bridge circuit shown in Fig. 3. It can be seen that the Pt100 value is obtained by computing the current ratio $I_{2} / I_{1}$ of tow bridge circuit loop. Eq. 6 not include the $E$ so reducing its fluctuations. At the same time it corrects the effect of lead resistance $r$ also. By Fig. 3 we have

$$
\begin{aligned}
& I_{2}=\frac{U_{\mathrm{s}}}{R_{2}} \\
& I_{1}=\frac{U_{p}}{R_{3}}
\end{aligned}
$$




$$
r=\frac{U_{r}-U_{p}}{I_{1}}=\frac{U_{r}-U_{p}}{U_{p}} R_{3}
$$

Put Eq. 7, Eq. 8, Eq. 9 into Eq. 6, we can get

$$
R_{t}=\left(R_{1}+R_{2}\right) \frac{R_{3}}{R_{2}} \frac{U_{\mathrm{s}}}{U_{p}}-2 R_{3} \frac{U_{r}-U_{p}}{U_{p}}-R_{3}
$$

In this way, after $R_{1}, R_{2}, R_{3}$ selected and sampling the voltage signal $U_{\mathrm{s}}, U_{r}, U_{p}$, the accurate value of Pt100 can be calculated by Eq. 10. That need not do any nonlinear compensation.

\section{Platinum Resistance Data Processing Circuit}

The platinum resistance signal processing circuit is shown in Fig. 4. It consists of the precision power supply $E$, bridge circuit, multi-channel switches M2, amplifier etc. MC1403 is used as precision voltage source $E$ and its output reference voltage is $2.5 \mathrm{~V} \pm 25 \mathrm{mV}$. here we take $E=2.525 \mathrm{~V}$ and its largest output current is $10 \mathrm{~mA}$. A intermittent power supply way is used for the bridge circuit to minimize the influence of electro-thermal effect when the Pt100 is powered on long time. The Pt100

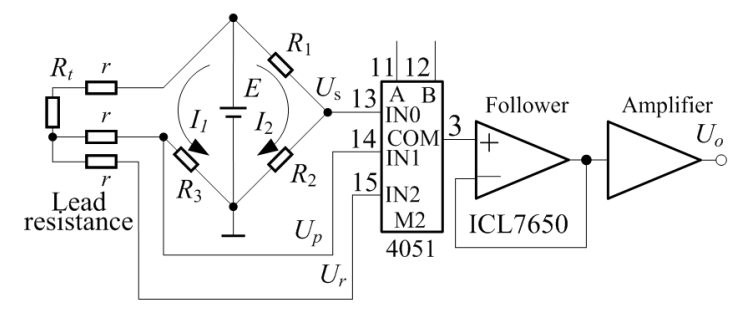

Figure 4 Platinum resistance data processing circuit

temperature range is $-200 \sim 850^{\circ} \mathrm{C}$, its resistance range is $18.52 \Omega \sim 390.48 \Omega, \Delta R_{t}=371.96 \Omega$. The manganin resistors are used for other resistors of bridge circuit in order to obtain stable temperature characteristic and precision resistance value. In order to let the $U_{p}$ having sufficient range where Pt100 value changing, and limit the bridge circuit current within $10 \mathrm{~mA}$, let $R_{1}=18 \Omega, R_{2}=R_{3}=R=500 \Omega$, so from the Eq. 10 we can get

$$
R_{t}=518 \frac{U_{\mathrm{s}}}{U_{p}}-1000 \frac{U_{r}-U_{p}}{U_{p}}-500
$$

Eq. 11 is the precise computing equation to calculate the $R_{t}$ with the measured voltage signal $U_{\mathrm{s},} U_{r}, U_{p}$. Let $r=0 \Omega, U_{\mathrm{s}}=2.437 \mathrm{~V}$, Eq. 11 as follow

$$
R_{t}=518 \frac{2.437}{U_{p}}-500=\frac{1262.5}{U_{p}}-500
$$

Now the Eq. 12 characteristic curve is shown in Fig. 5, it is a nonlinear curve.

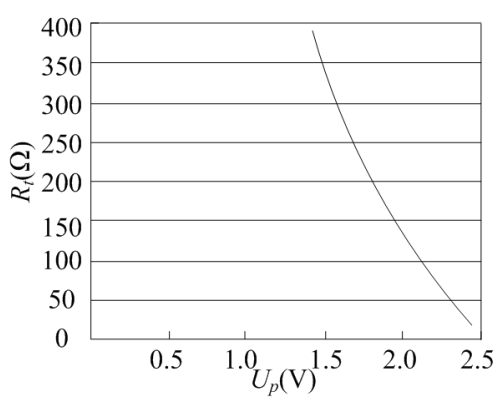

Figure 5. $R_{\mathrm{t}} \sim U_{\mathrm{p}}$ curve

From Fig. $4, U_{\mathrm{s}}$ is

$$
U_{\mathrm{s}}=\frac{R_{2}}{R_{1}+R_{2}} E=\frac{500}{18+500} 2.525 \approx 2.437 \mathrm{~V}
$$

Let $r=15 \Omega$, when $R_{t}=390.48 \Omega, U_{p}$ is smallest, its value is 


$$
U_{p \min }=\frac{R_{3}}{R_{t}+R_{3}+2 r} E=\frac{500}{390.48+500+2 \times 15} 2.525 \approx 1.372 \mathrm{~V}
$$

$U_{r}$ is smallest also, its value is

$$
U_{r \min }=\frac{R_{3}+r}{R_{t}+R_{3}+2 r} E=\frac{500+15}{390.48+500+2 \times 15} 2.525 \approx 1.413 \mathrm{~V}
$$

When $R_{t}=18.52 \Omega$, the $U_{p}$ is biggest, its value is

$$
U_{p \max }=\frac{R_{3}}{R_{t}+R_{3}+2 r} E=\frac{500}{18.52+500+2 \times 15} 2.525 \approx 2.302 \mathrm{~V}
$$

$U_{r}$ is biggest also, its value is

$$
U_{r \max }=\frac{R_{3}+r}{R_{t}+R_{3}+2 r} E=\frac{500+15}{18.52+500+2 \times 15} 2.525 \approx 2.371 \mathrm{~V}
$$

The maximum effective output of bridge circuit is

$$
\Delta U_{i \max }=U_{p \max }-U_{p \min }=0.93 \mathrm{~V}
$$

Voltage signal $U_{\mathrm{s}}, U_{r}, U_{p}$ be sent to the amplifier through multi-channel switch M2 time-sharing, the voltage amplification factor $K=4.1(\mathrm{~V} / \mathrm{V})$, so the $U_{\mathrm{s}}$ can be amplified to $10 \mathrm{~V}$, the $U_{r}$ and $U_{p}$ less than $10 \mathrm{~V}$, the amplifier output signals $U_{o}$ be sent to AD converter. $U_{\mathrm{s}}$ is partial voltage of $E$ on $R_{3}$ in the right side branch of the bridge circuit. Using sampling values in the Eq. 11 can play a role for power real-time self-calibration. The first level of amplifier adopts the follower in order to improve the input impedance and reduce the impact on the bridge circuit. here the follower is ICL7650, its input impedance is $10^{12} \Omega$, so the impact on the bridge circuit can be ignored completely.

\section{Structure of Platinum Resistance Bridge Thermometer}

The platinum resistance bridge thermometer, as shown in Fig. 6, consists of bridge circuit, multi-channel switch M1 and M2, amplifier, AD574, display, serial port, precision power supply $E$, $89 \mathrm{C} 51$ etc. The bridge circuit power supply $E$ is powered by the reference voltage chip MC1403. Under the control of the 89C51 I/O port P1.1, multi-channel switch M1 turn on during the data collection to supply $10 \mathrm{~V}$ to the chip MC1403 and MC1403 output $2.5 \mathrm{~V}$ reference voltage as power

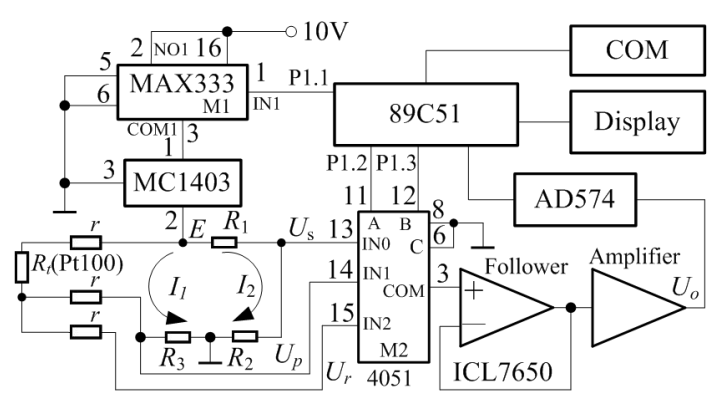

Fig. 6 The platinum resistance bridge thermometer structure diagram

supply $E$. After the data collection M1 turn off, MC1403 no output, no current flows through the Pt100, so the influence of electro-thermal effect on the measurement accuracy is reduced. The channel IN0, IN1 and IN2 of multi-channel switch M2 turn on time-sharing under the control of 89C51 I/O port P1.2, P1.3, sampling $U_{s}, U_{r}, U_{p}$ respectively, calculating the value of Pt100 by Eq. 11, and then looking into the Pt100 reference table to obtain the measured temperature. So the linear temperature measurement is implemented ${ }^{[6,7]}$. From the Eq. 18 we can get the maximum digital signal range $D_{\max }$ as

$$
D_{\max }=\frac{0.93}{2.437} \cdot 4096=1563
$$

The average resolution is $0.67^{\circ} \mathrm{C}$ in $-200 \sim 850^{\circ} \mathrm{C}$. The minimum resolution is $0.74^{\circ} \mathrm{C}$ at $850^{\circ} \mathrm{Cdue}$ to the nonlinear, as shown in Fig. 5. Considering the calculating rounding error, look-up reference table error and the influence of the lead resistance, the measurement error is not more than $1.5^{\circ} \mathrm{C}$ without considering the error of the Pt100 itself. The sampling period is $2 \mathrm{~s}$. 
There are 1051 temperature dividing points in Pt100 reference table in $-200 \sim 850{ }^{\circ} \mathrm{C} .10$ milliohm is used as the unit for each dividing point value of Pt100. The value is converted into hexadecimal number and stored in tow memory cells. The assembly Pt100 reference table is shown in Fig. 7. The

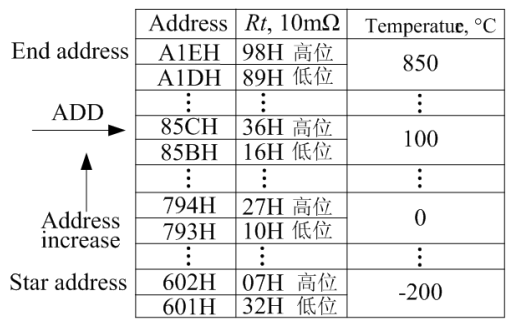

Fig.7 The assembly reference table diagram

assembly reference table occupying 2102 bytes and stored behind system programming. According to the Pt100 value calculated by Eq. 11 we can look into the assembly reference table to get its address. We use the high byte address and let it as ADD shown as Fig. 7. The temperature $t$ can be get as

$$
t=\frac{\mathrm{ADD}-602}{2}-200{ }^{\circ} \mathrm{C}
$$

The look-up table rounding error is less than $0.5^{\circ} \mathrm{C}$.

\section{Conclusions}

It is a important research topic in the temperature measurement field to implement the linear temperature measurement with platinum resistance. Many nonlinear compensation method is used in temperature measurement ${ }^{[1-5]}$. In the platinum resistance bridge thermometer a bridge circuit is used, the accurate measurement of the platinum resistance value is implemented by computing the current ratio of tow bridge circuit loop and then looking into the Pt100 reference table to get the measured temperature. The linear temperature measurement is realized without any nonlinear compensation. The measurement error is less than $1.5^{\circ} \mathrm{C}$. It is a very practical linear temperature measuring method. The measurement accuracy can be further improved if using high resolution $\mathrm{AD}$ or $0.1^{\circ} \mathrm{C} \mathrm{Pt} 100$ reference table.

\section{References}

[1] Bill Earlie, Platinum resistance thermometers[J],Control Engineering, 14 November 2009

[2] ZHENG Bao, CHENG Peng-zhan, ZHANG Jian-lan, Method of Improving the Measurement Accuracy of PRT[J], Metrology \& Measurement Technology, 2006-01, in Chinese

[3] AY Jiang, Y Tian, J Jiang.Experimental investigation method on improving the measurement accuracy of the platinum resistance thermometer[J], China Measurement Technology, 2007-05, in Chinese

[4]Chen Dixiang Pan Mengchun Meng Xianggui, Accurate temperature acquisition system[J], Electronic Measurement Technology, 2008-07,in Chinese

[5]WANG Long, Development of High-Precision Temperature Measurement and Control System Based on Pt100 Platinum Resistance[J], Journal of Jishou University(Natural Sciences Edition), 2009-03

[6]Liu Ximin, A Linear thermocouple temperature meter based on inverse reference function[C], 2010 ICICTA,Volume 1,IEEE

[7]Liu Xi-min, A Thermal-Resistor Temperature Measuring Device Based On Current Method[J], YIQIYIBIAOXUEBAO[J], 2007(4), v28.4, in Chinese 